\title{
सुगम संगीत में समाहित सांगीतिक तत्व
}

\author{
डॉ. उमा देवी
}

संगीत विभाग, हिमाचल प्रदेश विश्वविद्यालय, शिमला

\begin{abstract}
Indian Light Music is very popular in India. Geet, Gazal, Quwwali etc are included in the light music. This music has varieties of talas and layas. Composed in different raga light music touches the heart of music listeners'. Literature of light music is in Hindi, Urdu or local languages. Various instruments accompany the light music.
\end{abstract}

Key words : Light Music, Musical forms.

आधुनिक युग में सबसे ज़्यादा प्रचलित विधा सुगम संगीत ही है। इसकी लोकप्रियता का मुख्य कारण इसकी सुगमता है क्योंकि सुगम संगीत जन सामान्य के लिए आसानी से ग्राह्य है। इसके भाव जीवन से अधिक जुड़े हैं, अतः यह सर्वसाधारण के लिए अत्यधिक प्रभाव"ताली व आकर्षक प्रतीत होता है। सुगम संगीत में जितना आकर्षण इसके शब्दों से झलकता है उतना ही स्वरों से इसकी सौंदर्यात्मकता को अलंकृत कर के और भी आकर्षक बनाया जा सकता है। सुगम संगीत दो शब्दों का योग है :- 'सु'+'गम्' 'सु' का अर्थ है आसानी से और 'गम्' का अर्थ है ग्रहण किया जाना। अतः सुगम का अर्थ हुआ जिसका सम्पादन या साधन आसानी से या सुख पूर्वक किया जा सके।

सुगम संगीत शब्द प्रधान होने के साथ-साथ स्वर प्रधान भी है। इसमें शब्दों को स्वरों के माध्यम से सजीव बनाया जाता है और विभिन्न सांगीतिक तत्वों द्वारा शब्दों की अभिव्यक्ति को अलंकृत किया जाता है। सुगम संगीत में शब्द उसका शरीर है और स्वर उसकी आत्मा है एक दूसरे के बिना किसी का भी अस्तित्व नहीं है। किन्तु यहाँ एक बात ध्यान देने योग्य है कि इसकी अभिव्यक्ति अत्यन्त कठिन है क्योंकि इसमें भावों को प्रकट करने के विभिन्न ढंग से जिस प्रकार सांगीतिक तत्वों का प्रयोग किया जाता है उसको अभिव्यक्त करना बहुत कठिन है जिसके लिए संगीत की विधिवत् f"क्षा लेना ज़रूरी है। भावों के प्रस्तुतिकरण के लिए संगीत व शब्दों का प्रभाव स्वरों से प्रस्तुत करना यह एक आम व्यक्ति के द्वारा असम्भव है। कई बार किसी आवाज़ में मिठास व सांगीतिक गुण प्राकृतिक रूप में भी विद्यमान रहते हैं फ़िर भी संगीत का ज्ञान बहुत आव"यक है।

सुगम संगीत की विधाओं जैसे गीत, गज़ल, भजन, कव्वाली व लोक गीतों के प्रस्तुतिकरण के लिए कई सांगीतिक तत्वों द्वारा उसे सौंदर्यात्मक रूप दिया जा सकता है। इसकी प्रत्येक शैली में पृथक-पृथक सांगीतिक वि"ोषताएं हैं एक ओर जहां शास्त्रीय संगीत में स्वर, राग, लय के शासत्रीय नियमों की निबद्धता हैं वहीं दूसरी ओर सुगम संगीत की विधाओं में सांगीतिक तत्वों का प्रयोग सरल, स्वच्छन्द व चपलता के साथ होता है जिससे प्रत्येक शेली की पहचान अलग हो जाती है। सुगम संगीत में निम्नलिखित सांगीतिक तत्वों का प्रयोग होता है- 
1 रागतत्व - सुगम संगीत की प्रत्येक विधा की रचना का आधार रागदारी है। गीत, गज़ल, भजन, कव्वाली के शब्दों के अनुसार राग का चयन किया जाता है और बोलों के अनुसार राग चयन कर एक आधार"ीला रखी जाती है। गीतों में राग को मिश्रित करके उसमें चमत्कार उत्पन्न करने के लिए प्रयोग किया जाता है। बोलों के अनुसार राग के स्वरों के साथ-साथ अन्य राग के स्वर भी भावोत्पन्न करने के लिए लगाए जाते हैं। गज़ल का साहित्य थोड़ा कठिन होता है किन्तु गज़ल की रचना के लिए भी राग को आधार बनाया जाता है। ग़ज़ल के प्रत्येक शेर की विषय वस्तु अलग होती है। इसलिए भाव पैदा करने हेतु कई रागों का मिश्रण किया जाता है। कई बार एक ही शब्द प्रबल बनाने हेतु स्वरों को विभिन्न स्वर समूहों द्वारा वैचित्रय उत्पन्न करके आकर्षक बनाया जाता है। कई गज़लें एक ही राग पर आधारित होती है। उदाहरणार्थ उस्ताद मैंहदी हसन जी की एक म" हूर ग़ज़ल "गो जरा सी बात पर बरसों के याराने गए" राग अहीरी तोड़ी पर ही आधारित है। इसी प्रकार कव्वाली गायन विधा में भी राग पर आधारित रचनाएं सूफ़ी गायकों द्वारा गाई जाती रहीं हैं। कव्वाली के प्रारम्भ में राग वाचक आलाप गायन करके विभिन्न सूफ़ी पदों का गायन करके कव्वाली गायन किया जाता है। क़्वाल को और आकर्षित बनाने के लिए छोटी-छोटी तानों का प्रयोग भी कई बार युक्ति संगत लगता है। शब्दानुसार आविर्भाव-तिरोभाव द्वारा भी भावोत्पति की जाती है। हज़रत अमीर खुसरो द्वारा कई कव्वालियों की रचना की गई है। जो परम्परागत पीढ़ी दर पीढ़ी सिखाई जाती है। भजन गायन विधा में बंदि"। राग पर आधारित रहती है क्योंकि इसके भाव भक्तिप्रद होते हैं इसलिए स्वर प्रयोग सरल ढ़ग से किया जाता है। सुगम संगीत में सरल रागों जैसे :- खमाज, पहाड़ी, भिन्न 'ड्ज्ज, पीलू, यमन, विहाग, चारूके" शत्यादि राग प्रयोग किए जाते हैं।

2 ताल व लय - सुगम संगीत की शैलियों में सरल एवं छोटी तालों का प्रयोग किया जाता है। गीतों के लिए प्रायः दादरा, कहरवा, रूपक आदि तालों का प्रयोग होता है। लय को विषयानुसार कम-ज़्यादा रखा जाता है अर्थात् नृत्य गीत के लिए लय द्रुत रखी जाती है। प्रेम प्रणय गीत जैसे गीत मध्य लय में रखे जाते हैं। ग़ज़ में लय मध्य तथा तालों में कहरवा, दादरा, रूपक तथा कई बार झपताल का भी प्रयोग देखा गया है। भजन में भी मध्यलय तथा भजन का ठेका या अध्धा ताल का प्रयोग अत्याधिक किया जाता है इसके अलावा रूपक, दादरा, कहरवा व कई बार तीन ताल में भी भजन की रचनाएं की जाती है। कव्वाली में कव्वाली ताल का अत्यधिक प्रचलन है तथा कव्वाली गायन के लिए लय प्रायः द्रुत प्रयोग होती है। दादरा, कहरवा व रूपक में भी कव्वालियों की रचनाएं की गई हैं। कई सधे हुए कलाकारों द्वारा इसमें विभिन्न लयकारियों द्वारा बड़े ही सौंदर्य से प्रस्तुतिकरण किया जाता है।

3 सुगम संगीत में अलंकारिक तत्व - सुगम संगीत की विधाओं का गायन करते समय संगीत के विभिन्न अलंकरणों द्वारा इसे सौंदर्यात्मक रूप दिया जाता है। मुर्की, खटका, कण, गमक, मींड, तानें व अलाप जैसे संगीत के सुंदर उपकरणों द्वारा प्रत्येक विधा को और आकर्षक बनाया जा सकता है। गीतों के गायन में इसे और सुन्दर बनाने हेतु गायक अपनी ओर से निम"चत जगह व शब्द की जगह बड़ी चंचलता से खटका, मुर्की, व कण का प्रयोग करता है। परन्तु गीत के शब्द व स्वर समूहों में संतुलन बनाए रखना अत्यन्त आव"यक है इसी के साथ इन अलंकारिक तत्वों का आव"यकता से ज़्यादा प्रयोग गीत रचना को नीरस 
बना सकता है। ग़ज़ल गायन शैली में भाषा शेली के कठिन होने के कारण सर्वप्रथम इसके उच्चारण पर ध्यान देना आव"यक है इसी के साथ-साथ शब्दों का प्रभाव स्वरों द्वारा बरकरार रहे इसलिए आलाप, कण, खटका, मुर्की जैसे तत्वों को सुन्दरता से आव"यकतानुसार प्रयोग करना चाहिए इसके लिए अत्याधिक रियाज़ की आव"यकता होती है। इसी प्रकार क़्वाली गायन शैली में भी मुर्की, खटका, कण आदि तत्व सौंदर्य के लिए प्रयुक्त होते हैं।

कव्वाली गायन में लय द्रुत रहती है अतः इन तत्वों का प्रयोग बड़ी सावधानी से तथा तैयारी से करना पड़ता है। छोटी-छोटी क्षिप तानें, आलाप, मींड से भी भावाभिव्यक्ति तथा सौंदर्यता उत्पन्न की जाती है। कृव्वाली गायन शैली सामूहिक गायन शैली है अतः इसके मुखड़े व किसी भी पंक्ति का प्रभाव दिखाने के लिए एक शब्द को विभिन्न स्वर समूहों द्वारा अलग-अलग भी गाया जाता है। तालियां भी इसके गायन के साथ इसे और प्रभाव"ताली बनाती है तथा इसे अन्य विधाओं से अलग करती है।

भजन गायन शैली में भक्ति रस को बरकरार रखने हेतु इन्ही सांगीतिक तत्वों का प्रयोग सरल ढ़ग से किया जाता है। एक शब्द का प्रभाव दिखाने के लिए अलग-अलग स्वर समूहों द्वारा शब्द का उच्चारण किया जाता है। भजन गायन शैली की स्वर रचना में इसके शब्द तथा स्वर को यौगिक रूप इस प्रकार दिया जाता है कि भक्ति पदों से भक्ति रस बरबस ही उत्पन्न हो। कण, खटका, मुर्की जैसे तत्व केवल नाम मात्र ही प्रयोग हो तो ही अच्छा है अन्यथा शब्दों के भाव व भक्ति रस में प्रभाव का अल्पत्व पाया जाता है।

4 वाद्य यन्त्र - सुगम संगीत की विधाओं को लोकप्रियता दिलाने में विभिन्न वाद्य यंत्रों की भी महत्वपूर्ण भूमिका है इन वाद्य यन्त्रों के प्रयोग से किसी भी विधा को और भी आकर्षक बनाया जा सकता है। किन्तु इन वाद्य यन्त्रों का सही चयन यदि विधा के अनुसार किया जाए तो उसका प्रस्तुतिकरण और भी प्रभाव" $ा ल ी$ बनाया जा सकता है। गीतों के लिए उसकी आव"यकतानुसार व भावों को देखकर वाद्य यन्त्रों के का चयन किया जाता है। चित्रपट संगीत में एक गीत को तैयार करने के लिए जितना योगदान कम्पोज़र, लेखक, गायक, व निर्दे" क का होता है उतना ही योगदान संगीत संयोजक का भी होता है। जो दृ"य के अनुसार वाद्य यन्त्रों का प्रयोग करके उस दृ"य को तथा गीत को सजीव बनाता है। गीतों के लिए प्रायः हारमोनियम, सिन्थेसाइज़र, पियानों, एकॉर्डियन, ब्रास, सेक्सोफोन, बैन्जो, मैंडोलियन, बांसुरी, तानपुरा, सारंगी, सन्तूर, दिलरूबा, सितार, गिटार का प्रयोग किया जाता है। ताल वाद्यों में तबला, ढोलक, ऑक्टोपैड, कॉन्गो मृदंग, पखावज आदि का प्रयोग आव"यकतानुसार किया जा सकता है।

गज़ल गायन शब्द प्रधान गायकी है अतः इसके गायन के साथ कम वाद्य यन्त्रों का प्रयोग उचित प्रतीत होता है जैसे हारमोनियम, तबला, तानपुरा, वायलिन या सारंगी का वादन प्रभाव" ताली लगता है किन्तु रिकॉर्डिंग के दौरान कई अन्य प्रचलित वाद्य यन्त्रों जैसे गिटार, सिन्थीसाइज़र, पियानों इत्यादि का प्रयोग किया जाता है। कव्वाली गायन के साथ भी हारमोनियम, तबला, ढोलक का प्रयोग किया जा सकता है यह भी एक भक्तिमय गायन शैली ही है इन्हीं वाद्य यन्त्रों से इसका प्रभाव दिखाया जा सकता है। भजन के साथ तानपुरा, हारमोनियम, तबला करताल, घुंघरू, मंजीरा, डफली, चिमटा का प्रयोग किया जा सकता है। 
वास्तविकता हेतु इन वाद्य यन्त्रों के साथ भजन की स्वर रचना के भाव पक्ष को वास्तविकता प्रदान करके कलात्मकता व भक्ति रस संभव है:

इन्हीं वाद्य यन्त्रों से वृंद-वादन एवं अलग-अलग वादन कर गीत, ग़ज़ल, भजन, क़्वाली विधाओं से भावाभिव्यक्ति प्रदान करने का यत्न एवं कलात्मक पक्ष से शब्द व स्वरों से वास्तविकता लाने का यत्न किया जाता है।

5 हारमनी और मेलॉडी - गीतो में स्वर रचना को सरल तरीके से गाया जाता है। शब्दों के अनुसार स्वरों को ऊंचा-नीचा, सीधा या काकू तत्व द्वारा भावों को उकेरने के लिए प्रयोग होता है। और अधिक प्रभाव"ाली बनाने के लिए कभी हारमनी का प्रयोग किया जाता है। मुख्यतः समूह गीत में हारमनी का प्रयोग बहुत ही सुन्दर प्रतीत होता है। कुछ गायकों द्वारा मेलॉडी में स्वर रचना ज्यों की त्यों गाई जाती है तथा कुछ गायक इसी स्वर रचना को उसके सम्वादी स्वर को 'सा' मान कर गाते हैं। इसी प्रकार यही प्रकिया गायन-वादन के सम्वाद से भी सम्भव हो सकती है। सुगम संगीत की अन्य विधाओं में हारमनी का प्रयोग सम्भव नहीं है किन्तु वादन द्वारा किसी गायक के साथ स्वर रचना के संयोग से हारमनी सम्भव है।

निष्कर्ष सुगम संगीत सुनने में जितना सुगम है उतनी ही कठिन इसकी अभिव्यक्ति है। संगीत के सौंदर्यात्मक गुणों से सुसज्जित इसकी प्रत्येक शेली का अपना ही रंग अपना ही रूप व वि" ोषता है। इसकी शैलियों में जितना प्रभाव शब्दों का रहता है उतना ही इसके स्वरों की संगति का सम्वाद एक अलग छटा बिखेरता है।

इसकी शैलियों में राग, तान आलाप, कण, खटका, मुर्की, गमक से स्वरों को सजीव बना कर भावोत्पन्न करना इसकी लोकप्रियता का मुख्य कारण है। गीत, ग़ज़ल, भजन, कव्वाली में साहित्य के अनुरूप स्वर रचना को सांगीतिक तत्वों से सुसज्जित करके अपने-अपने भावों को उत्पन्न किया जाता है। जितना योगदान शब्दों व स्वरों के सम्वाद का है उतना ही योगदान वाद्य यन्त्रों की संगति का भी इसे लोकप्रियता प्रदान करने में है। स्वर रचनाकार भी इस लोकप्रियता के लिए दावेदार है। उसके बाद गायक-वादक का महत्वपूर्ण योगदान है क्योंकि शुरू से अंत तक का गायन-वादन इन कलाकारों पर निर्भर करता है कि वह प्रत्येक शैली में किस तरह इन सांगीतिक तत्वों से भाव को स्वरों द्वारा निभा पाता है। अन्ततः संगीत के तत्वों को साक्षात रूप देने हेतु संगीत की विधिवत् $f^{x}$ क्षा लेना सुगम संगीत के प्रस्तुतिकरण के लिए अत्यन्त आव"यक है।

\section{सन्दर्भ ग्रन्थ सूची}

भंडारी डॉ. प्रेम (1989) हिन्दुस्तानी संगीत में ग़ज़ल गायकी, राधा पब्लिकेशन्स, नई दिल्ली। अखलाक हुसैन देहलवी (1986), फ़न-ए शायरी, साहित्य रत्नालय, कानपुर।

आचार्य बृहस्पति (1977) संगीत चिंतामणी, कनिष्क पब्लिकेशन्स डिस्ट्रीब्यूटर्स, नई दिल्ली।

डॉ. विमल (2005) हिन्दी चित्रपट एवं संगीत का इतिहास, सोमनाथ दल, संजय प्रकाशन, नई दिल्ली। पैन्तल डॉ. गीता, डॉ. गीता (2009), पंजाब की संगीत परम्परा, राधा पब्लिकेशन्स, दिल्ली।

\section{पत्रिकाएँ}

भक्ति अंक, संगीत कार्यालय हाथरस उ.प्र., जनवरी 1970 ।

ग़ज़ल अंक, संगीत कार्यालय हाथरस उ.प्र., जनवरी 1976 । 